\title{
MULTIPLIERS VANISHING AT INFINITY FOR CERTAIN COMPACT GROUPS ${ }^{1}$
}

\section{ALESSANDRO FIGÀ-TALAMANCA}

ABSTRACT. We prove for certain compact groups $G$ and $1<p<\infty$, $p \neq 2$, that there exist operators commuting with left translations on $L^{p}(G)$ which are compact as operators on $L^{2}(G)$ but not as operators on $L^{p}(G)$.

Let $G$ be a compact group and let $\Gamma$ be the dual object of $G$, that is, the set of equivalence classes of irreducible unitary representations of $G$. For each $\gamma \in \Gamma$ we fix a representative $D_{\gamma}$ of $\gamma$ and a Hilbert space $\mathscr{S}_{\gamma}$ of dimension $d_{\gamma}$ on which $D_{\gamma}(x)$ acts. With this notation, if $f \in L^{1}(G)$ we can write the Fourier series of $f$ as

$$
f(x) \sim \sum_{\gamma \in \mathbf{\Gamma}} d_{\gamma} \operatorname{tr}\left(\hat{f}(\gamma) D_{\gamma}(x)\right)
$$

where $\operatorname{tr}$ is the ordinary trace and

$$
\hat{f}(\gamma)=\int_{G} f(x) D_{\gamma}\left(x^{-1}\right) d x
$$

is a linear transformation acting on $\mathscr{F}_{\gamma}$. (Warning: the notation here is not the same as in [7]: $\hat{f}(\gamma)$ denotes in this paper what Hewitt and Ross call the coefficient operator, cf. $[7,(34.3)(a)]$.)

Following [7] we denote by $\mathscr{F}=\mathfrak{S}(\Gamma)$ the space consisting those of functions $W$ on $\Gamma$ such that $W(\gamma)$ is a linear transformation on $\mathscr{F}_{\gamma}$ for each $\gamma \epsilon$ $\Gamma$.

Definition 1. An element $w \in \notin$ is called a multiplier of $L^{p}(G)(1 \leq p$ $\leq \infty)$ if for every $f \in L^{p}(G)$ the series $\Sigma_{\gamma \in \Gamma} d_{\gamma} \operatorname{tr}\left(W(\gamma) \hat{f}(\gamma) D_{\gamma}(x)\right)$, is the Four ier series of an element $T_{W} f$ of $L^{p}(G)$. The space of multipliers is denoted by $M_{p}(G)$.

We notice that the operators $T_{W}$ of $L^{p}$ into $L^{p}$ are linear and (by the closed graph theorem) continuous. We can endow therefore $M_{p}(G)$ with a Banach space norm: the norm of $W \in M_{p}(G)$ is defined to be the norm of the

Received by the editors January 30, 1973.

AMS (MOS) subject classifications (1970). Primary 43A15, 43A75, 22 C05.

1 Work supported by the C.N.R. 
corresponding operator $T_{W}$ on $L^{p}(G)$. It is easy to verify, for $p<\infty$, that the operators $T_{W}$, with $W \in M_{p}$ are exactly the bounded linear operators on $L^{p}(G)$ which commute by left translations.

Definition 2. A multiplier $W \in M_{p}(G)$ is said to vanish at infinity if $\lim _{\gamma \rightarrow \infty}\|W(\gamma)\|=0$, where the norm is that of $W(\gamma)$ as an operator on $\mathbb{S}_{\gamma}$.

It is known that if $G$ is an Abelian group and $p \neq 2$, there exist multipliers which vanish at infinity and are not the limit, in the norm of $M_{p}$, of multipliers with finite support. For $p=1$ or $p=\infty$ this is equivalent to the classical result which asserts the existence of singular measures with Fourier-Stieltjes transform vanishing at infinity. For $1<p<\infty, p \neq 2$, this was proved in [4].

We remark that multipliers which are the limit in $M_{p}(G)$ of finitely supported ones are precisely those for which the corresponding operator on $L^{p}(G)$ is compact and that the elements of $\mathscr{S}(\Gamma)$ which vanish at infinity correspond to compact operators on $L^{2}(G)$.

The purpose of the present paper is to extend the results described above, when $1<p<\infty$ and $p \neq 2$, to a class of noncommutative compact groups. We shall prove in fact the following:

Theorem A. Let $J$ be an infinite index set and let $G=\Pi_{i \in J} G_{i}$, where for each $i, G_{i}$ is a nontrivial compact group. Let $1<p<\infty$ and $p \neq 2$. Then there exists a multiplier $W \in M_{p}(G)$ which vanishes at infinity and is not the limit, in the norm of $M_{p}$, of finitely supported elements of $\mathbb{F}$.

The proof of this theorem is based on two lemmas. The first lemma is due to C. Fefferman and H. S. Shapiro [2, Theorem 1] and the second to A. Bonami [1, pp. 374-375]. Both lemmas were stated and proved only for commutative compact groups, but proofs can be easily translated into the language of noncommutative groups, as will be indicated below.

Lemma 1 (Fefferman and Shapiro). Let $1<p<\infty$, then there exists $a$ constant $a=\alpha(p)>0$ such that if $W \in M_{p}(G)$, and $W$ satisfies the conditions: (i) $W\left(\gamma_{0}\right)=0$, where $\gamma_{0}$ is the equivalence class of the trivial repre. sentation, (ii) $\|W\|_{M_{p}} \leq a(p)$; then the multiplier defined by $W^{\prime}\left(\gamma_{0}\right)=I$ (the identity operator), and $W^{\prime}(\gamma)=W(\gamma)$ for $\gamma \neq \gamma_{0}$, has norm one.

The proof of this lemma is almost exactly the same as that which is given in [2] for the corresponding result for commutative groups. Only two remarks are needed. First of all the norm-decreasing inclusion $M_{p}(G) \subseteq M_{2}(G)$, a well-known fact for $G$ commutative, is a consequence, for noncommutative 
$G$, of recent results of C. Herz [6, Theorem C]. Second, the proof of Theorem 1 in [2] makes use of the fact that $M_{p}=M_{q}$, for commutative $G$, when $1 / p+$ $1 / q=1$. This equality is not known to be true for noncommutative $G$, but we can use the known fact that $M_{p}=M_{q}^{\prime}$, where $M_{q}^{\prime}$ is the space of "right" multipliers of $L^{q}$, that is the space of $W \in\left(\right.$ such that for $f \in L^{q}$, the Fourseries $\Sigma_{\gamma \in \boldsymbol{\Gamma}} d_{\gamma} \operatorname{tr}\left(\hat{f}(\gamma) W(\gamma) D_{\gamma}(x)\right)$, represents a function in $L^{q}$ [3]. With these two remarks in mind the proof of Theorem 1 of [2] is easily reinterpreted to yield Lemma 1.

Before stating the second lemma we remark that if $G=G_{1} \times G_{2}$ where $G_{1}$ and $G_{2}$ are compact groups with dual objects $\Gamma_{1}$ and $\Gamma_{2}$, respectively, then the dual object $\Gamma$ of $G$ can be written as $\Gamma=\Gamma_{1} \times \Gamma_{2}$, in the sense that if $\gamma \in \Gamma$, there exists a unique pair $\left(\gamma_{1}, \gamma_{2}\right) \in \Gamma_{1} \times \Gamma_{2}$, such that any representative $D_{\gamma}$ of $\gamma$ is unitarily equivalent to the tensor product $D_{\gamma_{1}} \otimes$ $D_{\gamma_{2}}$ of a representative $D_{\gamma_{1}}$ of $\gamma_{1}$ and a representative $D_{\gamma_{2}}$ of $\gamma_{2}$. We shall then write $\gamma=\gamma_{1} \times \gamma_{2}[7$, Theorem 27.4.3].

Lemma 2 (A. Bonami). Let $G_{1}$ and $G_{2}$ be compact groups, with dual objects $\Gamma_{1}$ and $\Gamma_{2}$ and let $G=G_{1} \times G_{2}$. Let $W_{1}$ and $W_{2}$ be elements of $M_{p}\left(G_{1}\right)$ and $M_{p}\left(G_{2}\right)$, respectively, and suppose that $\left\|W_{1}\right\|_{M_{p}}=\left\|W_{2}\right\|_{M_{p}}=1$. (As before $\gamma_{0}$ denotes the class containing the trivial representation.) Then the element $W \in \mathcal{E}\left(\Gamma_{1} \times \Gamma_{2}\right)$ defined by $W(\gamma)=W_{1}\left(\gamma_{1}\right) \otimes W_{2}\left(\gamma_{2}\right)$, if $\gamma=\gamma_{1} \times$ $\gamma_{2}$, is an element of $M_{p}(G)$ and $\|W\|_{M_{p}} \leq 1$.

Again, the proof of this lemma is exactly as in the commutative case [1, Lemma 1, p. 375].

Proof of Theorem A. If $G=\Pi_{i \in J} G_{i}$ where $G_{i}$ are nontrivial groups and $J$ is an infinite set, we may assume, without loss of generality, that each $G_{i}$ is infinite (if not divide $J$ into infinitely many infinite subsets and group together the factors). We may also assume for simplicity that $J$ is countable and in fact that $J=\{1,2, \cdots\}$. For each $i$ we know that since $p \neq 2$ the norm-decreasing inclusion $M_{p}\left(G_{i}\right) \subseteq M_{2}\left(G_{i}\right)$ is strict [5, Theorem 6]. This implies that the norm of $M_{p}\left(G_{i}\right)$ is not equivalent to that of $M_{2}\left(G_{i}\right)$ because $M_{p}$ is not closed in $M_{2}$. Therefore we can find a finitely supported $W_{i} \in M_{p}\left(G_{i}\right)$ such that $W_{i}\left(\gamma_{0}\right)=0,\left\|W_{i}\right\|_{M_{2}} \leq 1 / i,\left\|W_{i}\right\|_{M_{p}}=\alpha(p)$, where $\alpha(p)$ is the constant appearing in the statement of Lemma 1 . Let $W_{i}^{\prime}$ be the multiplier satisfying $W_{i}^{\prime}\left(\gamma_{0}\right)=I, W_{i}^{\prime}(\gamma)=W_{i}(\gamma)$ for $\gamma \neq \gamma_{0}$, whose norm is one by Lemma 1 .

Applying inductively Lemma 2 we can construct elements $W^{(n)} \in M_{p}\left(G^{(n)}\right)$ where $G^{(n)}=\Pi_{i=1}^{n} G_{i}$, such that if $\Gamma^{(n)}$ is the dual object of $G^{(n)}$, and $\gamma \epsilon$ $\Gamma^{(n)}, \gamma=\gamma_{1} \times \cdots \times \gamma_{n}$, then $W^{(n)}(\gamma)=W_{1}^{\prime}\left(\gamma_{1}\right) \otimes \cdots \otimes W_{n}^{\prime}\left(\gamma_{n}\right)$ and $\left\|W^{(n)}\right\|_{M_{p}}$ 
$\leq 1$. Obviously we may consider $W^{(n)}$ as an element of $M_{p}(G)$ with the same norm, by defining $W^{(n)}(\gamma)=0$, if $\gamma \notin \Gamma_{1} \times \cdots \times \Gamma_{n}$, where $\Gamma_{i}$ is the dual object of $G_{i}$. Finally let $W$ be a weak* limit of $W^{(n)}$ (we consider $M_{p}$ as the dual space of $\left.A_{p}[3]\right)$. Then $W(\gamma)=W^{(n)}(\gamma)$ if $\gamma \in \Gamma_{1} \times \cdots \times \Gamma_{n}$. We must show that $W$ vanishes at infinity and is not the limit in the norm of $M_{p}$ of finitely supported multipliers. Let $\epsilon>0$ be given and let $1 / n<\epsilon$. Denote by $K_{n}$ the finite set $K_{n}=\left\{\gamma_{1} \times \cdots \times \gamma_{n}: \gamma_{i} \in \operatorname{supp} W_{i}^{\prime} \subseteq \Gamma_{i}\right\}$. Let $\gamma \notin K_{n}$ and suppose $W(\gamma) \neq 0$.

Let $\gamma=\gamma_{1} \times \cdots \times \gamma_{m}$; with $\gamma_{i} \in \Gamma_{i}$ and $\gamma_{m} \neq \gamma_{0}$. Since $\gamma \notin K_{n}$ and $W(\gamma) \neq 0$, then $m>n$. Now $W(\gamma)=W_{1}^{\prime}\left(\gamma_{1}\right) \otimes \cdots \otimes W_{m}^{\prime}\left(\gamma_{m}\right)$. Therefore $\|W(\gamma)\|$ $\leq\left\|W_{1}^{\prime}\left(\gamma_{1}\right)\right\| \cdots\left\|W_{m}^{\prime}\left(\gamma_{m}\right)\right\| \leq\left\|W_{m}^{\prime}(\gamma)\right\|$, but since $\gamma_{m} \neq \gamma_{0}, W_{m}^{\prime}\left(\gamma_{m}\right)=W_{m}\left(\gamma_{m}\right)$; therefore,

$$
\|W(\gamma)\| \leq\left\|W_{m}\left(\gamma_{m}\right)\right\| \leq \sup _{\gamma \in \Gamma_{m}}\left\|W_{m}(\gamma)\right\|=\left\|W_{m}\right\|_{M_{2}}<1 / n .
$$

We have proved that if $\gamma \in K_{n},\|W(\gamma)\|<1 / n$ and hence $W$ vanishes at

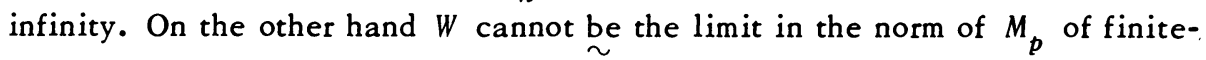
ly supported multipliers, for suppose $\widetilde{W}$ is a finitely supported multiplier satisfying $\|\widetilde{W}-W\|_{M_{p}}<a(p) / 4$. Since the support of $\widetilde{W}$ is finite, for some $m, \Gamma_{m} \cap \operatorname{supp} \tilde{W} \subseteq\left\{\gamma_{0}\right\}$.

Now $\left\|W_{m}\right\|_{M_{p}}>a(p) / 2$ so there exists a trigonometric polynomial $f$ with $\|f\|_{p}=1, \operatorname{supp} \hat{f} \subseteq \Gamma_{m}$ such that $\left\|T_{W_{m}} f\right\|_{p}>\alpha(p) / 2$.

Let $E \in \mathbb{E}(\Gamma)$ denote the characteristic function of $\gamma_{0}$. Then $E \in M_{p}(G)$, $\|E\|_{M_{p}}=1$, and $E+W_{m}=W_{m}^{\prime}$. Also $W \mid \Gamma_{m}=W_{m}^{\prime}$ and $W\left(\gamma_{0}\right)=1$. Thus one verifies that

$$
\left(T_{W}-T_{\widetilde{W}}\right) f=T_{E}\left(T_{W}-T_{\widetilde{W}}\right) f+T_{W} f
$$

Therefore

$$
\begin{aligned}
\frac{\alpha(p)}{4} & >\|W-\widetilde{W}\|_{M_{p}} \geq\left\|\left(T_{W}-T_{\widetilde{W}}\right) f\right\|_{p} \\
& \geq\left\|T_{W_{m}} f\right\|_{p}-\left\|T_{E}\left(T_{W}-T_{\widetilde{W}}\right) f\right\|_{p} \\
& >\alpha(p) / 2-\alpha(p) / 4=\alpha(p) / 4
\end{aligned}
$$

a contradiction. This completes the proof of the Theorem.

\section{REFERENCES}

1. A. Bonami, Étude des coefficients de Fourier des fonctions de $L^{p}(G)$, Ann. Inst. Fourier (Grenoble) 20 (1970), fasc. 2, 335-402. MR 44 \#727. 
2. C. Fefferman and H. S. Shapiro, A planar face on the unit sphere of the multiplier space $M_{p}, 1<p<\infty$, Proc. Amer. Math. Soc. 36 (1972), 435-439.

3. A. Figà-Talamanca, Translation invariant operators in $L^{p}$, Duke Math. J. 32 (1965), 495-501. MR 31 \#6095.

4. A. Figà-Talamanca and G. I. Gaudry, Multipliers of $L^{p}$ which vanish at infinity, J. Functional Analysis 7 ( 1971 ), 475-486. MR 43 \#2429.

5. A. Figà-Talamanca and D. Rider, $A$ theorem of Littlewood and lacunary series for compact groups, Pacific J. Math. 16 (1966), 505-514. MR 34 \#6444.

6. C. Herz, The theory of p-spaces with an application to convolution operators, Trans. Amer. Math. Soc. 154 (1971), 69-82. MR 42 \#7833.

7. E. Hewitt and K. Ross, Abstract harmonic analysis. Vol. II. Structure and análysis for compact groups analysis on locally compact Abelian groups, Die Grundlehren der math. Wissenschaften, Band 152, Springer-Verlag, New York and Berlin, 1970. MR $41 \# 7378$.

ISTITUTO MATEMATICO, UNIVERSITÀ DI GENOVA, 16132 GENOVA, ITALY 